\section{(2) \\ BRAZIILIAN JOURNAL \\ OF MEDICAL AND BIOLOGICAL RESEARCH}

www.bjournal.com.br
ISSN 0100-879X

Volume 45 (5) 376-472 May 2012

BIOMEDICAL SCIENCES

AND

CLINICAL INVESTIGATION

Braz J Med Biol Res, May 2012, Volume 45(5) 450-458

doi: 10.1590/S0100-879X2012007500025

Linear and nonlinear analysis of heart rate variability in healthy subjects and after acute myocardial infarction in patients

V.C. Kunz, E.N. Borges, R.C. Coelho, L.A. Gubolino, L.E.B. Martins and E. Silva

The Brazilian Journal of Medical and Biological Research is partially financed by

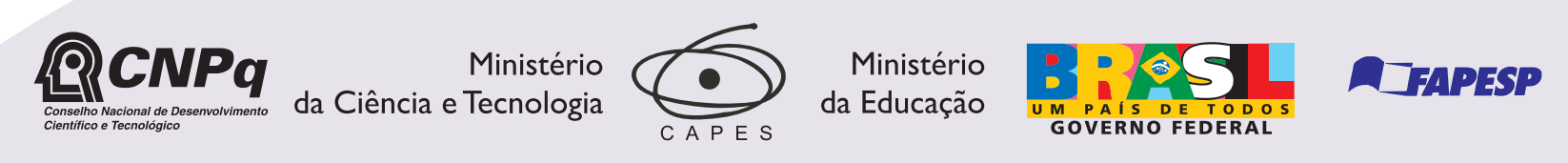

Institutional Sponsors

sciedo
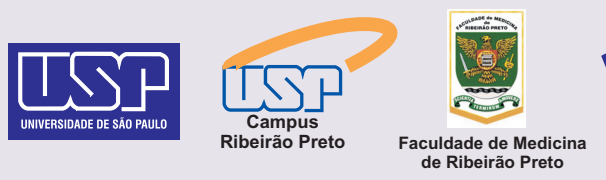

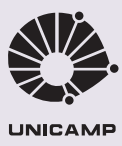

Ф SHIMADZu

Associaçăo
Fundo de Incentivo
à Pesquisa

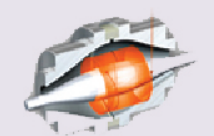

lore High - Performance MS Orbitrap Technology analitica Thermo 


\title{
Linear and nonlinear analysis of heart rate variability in healthy subjects and after acute myocardial infarction in patients
}

\author{
V.C. Kunz ${ }^{1}$, E.N. Borges ${ }^{2}$, R.C. Coelho ${ }^{3}$, L.A. Gubolino ${ }^{4}$, \\ L.E.B. Martins ${ }^{5}$ and E. Silva ${ }^{1,2}$ \\ ${ }^{1}$ Laboratório de Fisioterapia Cardiovascular, Núcleo de Pesquisa em Exercício Físico, \\ Departamento de Fisioterapia, Universidade Federal de São Carlos, São Carlos, SP, Brasil \\ ${ }^{2}$ Faculdade de Ciências da Saúde, Universidade Metodista de Piracicaba, Piracicaba, SP, Brasil \\ 3 Instituto de Ciência e Tecnologia, Universidade Federal de São Paulo, São José dos Campos, SP, Brasil \\ ${ }^{4}$ Unidade Coronariana do Hospital dos Fornecedores de Cana de Piracicaba, Piracicaba, SP, Brasil \\ ${ }^{5}$ Faculdade de Educação Física, Universidade Estadual de Campinas, Campinas, SP, Brasil
}

\begin{abstract}
The objectives of this study were to evaluate and compare the use of linear and nonlinear methods for analysis of heart rate variability (HRV) in healthy subjects and in patients after acute myocardial infarction (AMI). Heart rate (HR) was recorded for $15 \mathrm{~min}$ in the supine position in 10 patients with AMI taking $\beta$-blockers (aged $57 \pm 9$ years) and in 11 healthy subjects (aged 53 \pm 4 years). HRV was analyzed in the time domain (RMSSD and RMSM), the frequency domain using low- and high-frequency bands in normalized units (nu; LFnu and HFnu) and the LF/HF ratio and approximate entropy (ApEn) were determined. There was a correlation $(P<0.05)$ of RMSSD, RMSM, LFnu, HFnu, and the LF/HF ratio index with the ApEn of the AMI group on the 2nd $(r=0.87,0.65,0.72,0.72$, and 0.64$)$ and 7 th day $(r=0.88,0.70,0.69,0.69$, and 0.87$)$ and of the healthy group ( $r=0.63$, $0.71,0.63,0.63$, and 0.74 ), respectively. The median HRV indexes of the AMI group on the 2nd and 7th day differed from the healthy group $(P<0.05)$ : RMSSD $=10.37,19.95,24.81 ; \mathrm{RMSM}=23.47,31.96,43.79 ; \mathrm{LFnu}=0.79,0.79,0.62 ; \mathrm{HFnu}=0.20$, $0.20,0.37 ; \mathrm{LF} / \mathrm{HF}$ ratio $=3.87,3.94,1.65 ; \mathrm{ApEn}=1.01,1.24,1.31$, respectively. There was agreement between the methods, suggesting that these have the same power to evaluate autonomic modulation of HR in both AMI patients and healthy subjects. AMI contributed to a reduction in cardiac signal irregularity, higher sympathetic modulation and lower vagal modulation.
\end{abstract}

Key words: Myocardial infarction; Heart rate variability; Autonomic nervous system; Spectral analysis; Nonlinear dynamics

\section{Introduction}

The integrity of autonomic modulation of heart rate (HR) is evaluated by analyzing heart rate variability (HRV), which refers to oscillations in the intervals between consecutive heartbeats, or R-R intervals (RRi) (1). This method for evaluation is employed in a variety of clinical applications, being extensively used in cardiology due to its noninvasiveness, ease of application and high reproducibility $(1,2)$.

It has been well established that autonomic HR regulation is associated with the integrity of complex interactions between electrophysiological, humoral and hemodynamic variables $(2,3)$. However, HR is considered to present nonlinear behavior due to the complex interaction between several systems (central nervous system, reflex mechanisms and neurohumoral factors) (3-7).
The literature indicates that linear methods have been widely explored and are considered to be appropriate for the study of HRV (8-12). Nevertheless, interest in nonlinear methods has increased in recent years $(4-7,13-15)$ due to observations that HR fluctuation is subordinate to autonomic nervous system control of cardiac activity and vascular dynamics (14), which suggests that the mechanisms involved in cardiovascular regulation interact with and have repercussions on other organs.

Among nonlinear methods, approximate entropy (ApEn) allows the evaluation of regular time series and is used to analyze physiological and clinical data $(13,14)$. Thus ApEn provides quantitative information about the complexity of or reduction in the chaotic behavior of the signal of both

Correspondence: E. Silva, Universidade Federal de São Carlos, Rodovia Washington Luís, km 235, 13565-905 São Carlos, SP, Brasil. Fax: +55-16-3361-2081. E-mail: esilvas@unimep.br or vandenikunz@yahoo.com.br 
short-term and long-term data recordings (2-4,13-15). The application of ApEn to cardiac signal analysis provides information about the autonomic modulation of $H R$, considering that increased signal regularity is related to pathological situations such as acute myocardial infarction (AMI). A study that evaluated HRV for an average period of 30 days post-AMI without the use of $\beta$-blockers found no correlation between the indexes of linear and nonlinear methods. Moreover, in this same study greater irregularity in HR dynamics was observed in AMI patients than in healthy subjects (2).

Santos-Hiss et al. (10), using linear models to evaluate the short-term evolution of the autonomic modulation of HR in patients taking $\beta$-blockers and receiving routine clinical intervention, observed no modification of HRV indexes between the 1st and 6th day after AMI.

Thus, the hypothesis of the present study was that both the application of a nonlinear method like ApEn and linear methods in the time and frequency domains could be used to identify and evaluate the HR dynamics of both post-AMI patients who take $\beta$-blockers and healthy individuals. Additionally, post-AMI patients presented alterations in the dynamics of autonomic modulation of HR compared to healthy subjects.

The objectives of the present study were: 1) to evaluate the relationship between HRV analysis in the time and frequency domains with ApEn in both post-AMI patients taking $\beta$-blockers and healthy individuals; 2 ) to compare HRV indexes on the 2nd and 7th day after AMI; 3) to compare the HRV indexes between AMI and healthy groups.

\section{Material and Methods}

\section{Design and subjects}

Sixty-four men (36 with AMI and 28 healthy subjects) ranging in age from 50 and 65 years volunteered for the study through personal invitation or advertising. In this group, 10 men with AMI ( $57 \pm 9$ years old) and 11 healthy men (54 \pm 3 years old) were enrolled and completed the study. Figure 1 is a flow diagram presenting the sample loss in detail.

The AMI group was selected from two hospitals in the State of São Paulo, Brazil: the coronary units of Hospital dos Fornecedores de Cana in the city of Piracicaba and the Santa Casa de Misericórdia in the city of Limeira. Of the 36 volunteers who had been admitted to the coronary unit and diagnosed with AMI, 10 were included in the study. They had all been submitted to Doppler echocardiography and cardiac catheterization with chemical or mechanical reperfusion in the first hours after the diagnosis of $\mathrm{AMI}$, had been using $\beta$-blocking therapy (atenolol, $46 \pm 9.4 \mathrm{mg} /$ day), their left ventricular ejection fraction was within normal limits $(0.61 \pm 0.06)$, and their clinical classification was Killip class I. For the healthy group, 28 volunteers were evaluated and 11 who presented no evidence of cardiovascular, respiratory, musculoskeletal, and/or metabolic anomalies were included. Participants in this group were taking no medications and did not smoke or drink.

Volunteers from both groups were attended by the hospital medical team, undergoing clinical and cardiovascular evaluation as well as biochemical blood tests [total cholesterol and fractions (HDL, LDL), glycemia, triglycerides, creatinine, and uric acid].

The diagnosis of AMI was confirmed by the presence of two or more of the following criteria: 1) precordialgia or retrosternal pain, either constrictive or burning, whether radiating or not to the upper limbs, neck and back, that lasted $>30$ min and was not treated with vasodilators; 2 ) elevation of segment ST $>1 \mathrm{~mm}$ in at least two or more contiguous derivations in the electrocardiogram (ECG); 3) elevation of the myocardial necrosis markers creatine kinase-MB and creatine phosphokinase to twice the normal values (16).

This cross-sectional study was approved by the Ethics Committee of Universidade Metodista de Piracicaba (protocol \#63/06). The relevance and experimental procedures of the study were described to the volunteers, who signed a free informed consent form.

\section{Experimental procedures}

$\mathrm{HR}$ and blood pressure (BP) data from the AMI group were collected in the coronary units on the 2nd and 7th day after the coronary event, and data collection from the healthy group was performed at the institution's research laboratory. All protocols were carried out by the same researchers and in the morning to avoid circadian influence on the variables studied. The data collection environments were climate controlled, with temperature and relative humidity of approximately $23^{\circ} \mathrm{C}$ and $50 \%$, respectively. Volunteers were instructed to avoid caffeine, alcohol and strenuous exercise on the day before the protocol. On the day of the experiment, the volunteers were interviewed to determine whether they had followed the instructions and if the control conditions (HR and BP) were within the normal range. While in the supine position, subject BP was checked three times using the Korotkoff auscultatory method with a mercury-column sphygmomanometer (WanMed, Brazil) and a stethoscope (Littman, USA).

In order to reduce anxiety, the volunteers were familiarized with the equipment and the protocol before the experiment.

\section{Experimental protocol}

First, the subjects were kept resting in the supine position for $10 \mathrm{~min}$. Next, HR and RRi were recorded continuously for 15 min with a digital telemetry system consisting of a transmitter placed on the patient's chest and an HR monitor (Polar ${ }^{\circledR}$ S810i; Polar Electro, Oy, Finland). The system detects ventricular depolarization, corresponding to the $R$ wave on the ECG, with a sampling rate of 500 $\mathrm{Hz}$ and a temporal resolution of $1 \mathrm{~ms}$ (17), and has been previously validated by Loimaala et al. (18), Gamelin et al. 
Figure 1. Flowchart of sample loss.

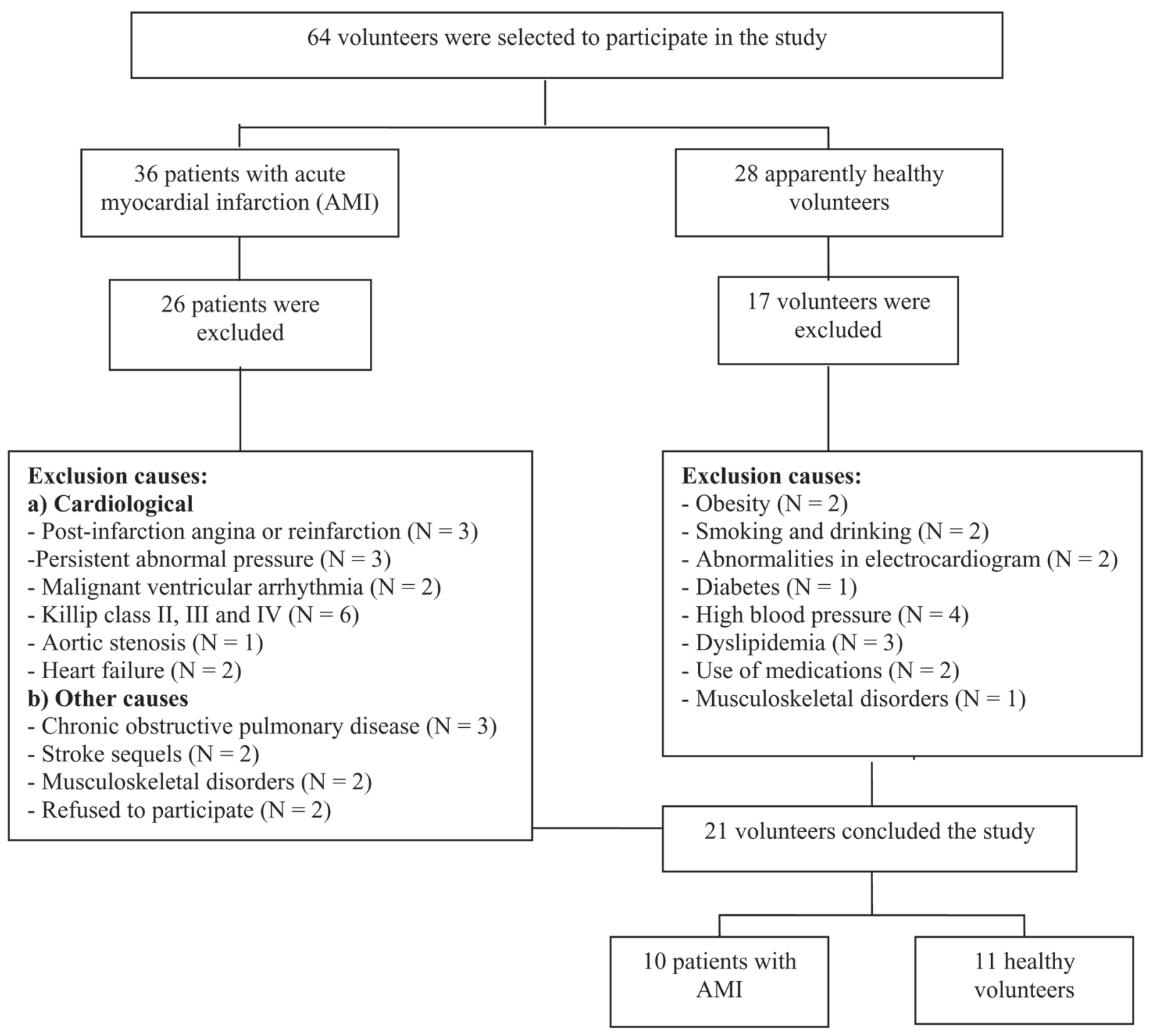

(19), and Vanderlei et al. (20). The RRi captured with the HR monitor can be analyzed with both linear and nonlinear models, including ApEn (21). After recording, the signals were transmitted to a receiver and interface connected to a computer for subsequent analysis.

\section{Data analysis}

Resting HR evaluation was based on the mean value of the 15-min assessment and BP evaluation was based on the mean value of BP measurements.

The first step in the data analysis involved a visual inspection of RRi (ms) distribution in the ECG in order to select the sections with the highest signal stability. Ectopic beats were excluded and 700 data points were used in the analysis.

HRV analysis was carried out using the following linear methods: A) in the time domain, RMSSD (square root of the mean of the sum of the squares of differences) between adjacent $\mathrm{RRi}$ divided by the number of $\mathrm{RRi}$ minus one, expressed in ms) and RMSM (square root of the sum of the squares of differences of individual values compared to the mean value, divided by the number of RRi in a period) (1); $B$ ) in the frequency domain, an autoregressive algorithm was used $(22,23)$. Using power spectrum components, very low frequency (VLF $=0.003$ at $0.04 \mathrm{~Hz}$ ), low frequency (LF $=0.04$ at $0.15 \mathrm{~Hz}$ ) and high frequency bands $(\mathrm{HF}=0.15$ at $0.4 \mathrm{~Hz}$ ) were obtained, as well as the ratio between absolute 
LF and HF areas (LF/HF ratio). Normalization was carried out by dividing the absolute power of a given component $\mathrm{ms}^{2}$ (LF or HF) by the total power spectrum, subtracting the VLF component and multiplying by 100 . HF and LF bands represent the action of vagal and sympathetic components in $\mathrm{HR}$ regulation, respectively (1).

The nonlinear method (ApEn) was carried out by the method of Pincus $(13,14)$. The basic idea of ApEn is to determine the correlation measure for blocks of $m$ samples of signal. Let $x_{i}$ and $x_{j}$ be any pair of blocks of size $m$ samples of $u(1), u(2), \ldots, u(N)$ signal, $N$ the number of signal samples, a positive integer $m(m \leq N)$ indicates the size of the bocks used for comparison, and a positive number integer $r$ sets the upper limit on the consideration of similarity between $x_{i}$ and $x_{j}$. Thus, it is possible to define the following correlation measure:

$C_{i}^{m}(r)=\frac{B_{i}}{(N-m+1)}$

where $B_{i}$ is the number of $x(j)$ blocks with a maximum distance of $x(i) \leq r$, i.e., number of $x(j)$ blocks such that $d[x(i)$, $x(j)] \leq r ;(N-m+1)$ is the total amount of vector of size $m$ in the signal.

The distance between two vectors can be calculated as:

$d[x(i), x(j)]=\max _{k=1, \ldots m}(\mu(i+k-1)-\mu(j+k-1))$

The following measure adds the contributions of the log of the correlations:

$\phi^{m}(r)=\frac{\sum_{i=1}^{N-m+1} \log C_{i}^{m}(r)}{N-m+1}$

where log represents the natural logarithm. The function calculates the average natural logarithm of $C_{i}^{m}(r)$ functions.

ApEn is then defined as:

$\operatorname{ApEn}(m, r)=\left\{\begin{array}{l}\lim _{N-\infty}\left\{\phi^{m}(r)-\phi^{m+1}(r)\right\}, m>0 \\ \lim _{N-\infty} \quad\left\{-\phi^{\dagger}(r)\right\}, \quad m=0\end{array}\right.$

Given $N$ samples of the signal, the ApEn is implemented by defining the following statistic:

$\operatorname{ApEn}(m, r, N)= \begin{cases}\Phi^{m}(r)-\Phi^{m+1}(r) & m>0 \\ -\Phi^{1}(r) \quad m=0 & \end{cases}$

In our experiments the following values were used for the variables: $m=2$ (size of the blocks used for comparison), $r=20 \%$ multiplied by the standard deviation of the signal (upper limit for considering data similarity), and $N=700$ (number of data points in the signal).

Data were analyzed with MATLAB 6.5 (R13) using the HRV analysis routine.

\section{Statistical analysis}

According to the frequency distribution analysis (Kolmogorov-Smirnov), the data were not normally distributed. In order to evaluate the relationship between the HRV analyses in the time and frequency domains and ApEn, a simple linear regression was applied to the AMI group on the 2 nd and 7 th day and to the healthy group. The Wilcoxon nonparametric test was used to compare the HRV data from the AMI group on the 2nd and 7th day after the coronary event. The MannWhitney nonparametric test was used to compare the HRV data between the AMI group and the healthy group. The level of significance was established at $5 \%$ for all analyses. Calculations were performed using the BioEstat 5.0 software (Brazil). A pre-study power analysis suggested that a minimum of 10 subjects in each group and a $5 \%$ level of significance would yield at least an $80 \%$ power.

\section{Results}

\section{Sample characteristics}

Table 1 presents the data for the AMI group and the healthy group regarding age, anthropometric characteristics, resting cardiovascular variables, and clinical characteristics, as well as risk factors for the AMI group. No significant differences were observed between groups.

\section{Analysis of HRV indexes}

In the linear regression analysis between time and frequency domains and ApEn indexes, there was a significant correlation between the AMI group on the 2nd and 7 th day after the coronary event and the healthy group (Table 2).

Figure 2A and B show that the RMSSD and RMSM $H R V$ indexes of the healthy group were higher $(P<0.05)$ than those of the AMI group on the 2 nd and 7 th day. The indexes of the AMI group were similar on the $2 \mathrm{nd}$ and 7 th day $(P>0.05)$. Frequency domain analysis revealed a higher LFnu (normalized unit) index (Figure 2C) and LF/ HF (Figure 2E) and a lower HFnu index (Figure 2D) in the AMI group on the 2nd and 7 th day compared to the healthy group $(P<0.05)$, indicating that the healthy group presented lower sympathetic modulation and higher vagal modulation than the AMI group. No differences were found in the intergroup comparison for these indexes $(P$ $>0.05)$. There were also no significant intergroup differences in the nonlinear HRV analysis based on ApEn (Figure 2E; $P>0.05$ ), although the healthy group values were higher $(P<0.05)$ than those of the AMI group on the 2 nd and 7 th day. 
Table 1. Age, anthropometric characteristics, cardiovascular variables, and clinical characteristics of the groups studied.

\begin{tabular}{lcr}
\hline & AMI group $(\mathrm{N}=10)$ & Healthy group $(\mathrm{N}=11)$ \\
\hline Age and anthropometric characteristics & & \\
Age $($ years $)$ & $57.10 \pm 9.22$ & $53.45 \pm 3.42$ \\
Height $(\mathrm{m})$ & $1.67 \pm 0.04$ & $1.69 \pm 0.05$ \\
Body mass $(\mathrm{kg})$ & $74.60 \pm 13.09$ & $79.59 \pm 8.34$ \\
Body mass index $\left(\mathrm{kg} / \mathrm{m}^{2}\right)$ & $26.81 \pm 4.22$ & $27.86 \pm 3.11$ \\
Cardiovascular variables & & \\
Heart rate $($ bpm) & $65.70 \pm 10.86$ & $70.18 \pm 9.58$ \\
Systolic blood pressure $(\mathrm{mmHg})$ & $126.00 \pm 13.49$ & $116.42 \pm 6.43$ \\
Diastolic blood pressure $(\mathrm{mmHg})$ & $76.00 \pm 10.74$ & $77.73 \pm 4.10$ \\
Clinical characteristics & & \\
Smoking & $5(50)$ & $0(0)$ \\
High blood pressure $\geq 140 / 90 \mathrm{mmHg}$ & $2(20)$ & $0(0)$ \\
Fasting hyperglycemia $\geq 126 \mathrm{mg} / \mathrm{dL}$ & $3(30)$ & $0(0)$ \\
Hypercholesterolemia $>240 \mathrm{mg} / \mathrm{dL}$ & $3(30)$ & $0(0)$ \\
Hypertriglyceridemia $>200 \mathrm{mg} / \mathrm{dL}$ & $4(40)$ & $0(0)$ \\
AMl location & & - \\
Anterior & $3(30)$ & - \\
Posteroinferior & $7(70)$ & - \\
Killip clinical class I & $10(100)$ & - \\
LVEF $>40 \%$ & $10(100)$ & - \\
Reperfusion & & - \\
Chemical & $4(20)$ & - \\
Mechanical & $6(60)$ & - \\
Medications & & \\
B-blockers & & \\
ACEl & & \\
\hline
\end{tabular}

Data are reported as means $\pm S D$, absolutes values and percentage. $\mathrm{AMI}=$ acute myocardial infarction; LVEF = left ventricular ejection fraction; ACEI = angiotensin-converting enzyme inhibitors. The Mann-Whitney test was used to compare age and anthropometric characteristics and cardiovascular variables between groups; there were no statistically significant differences.

Table 2. Correlation between approximate entropy (ApEn) and indexes in time and frequency domains of HRV (RMSSD, RMSM, LFnu, HFnu, and LF/HF ratio) for the groups studied.

\begin{tabular}{|c|c|c|c|c|c|c|c|c|c|}
\hline & \multicolumn{3}{|c|}{ AMI group, 2 nd day $(N=10)$} & \multicolumn{3}{|c|}{ AMI group, 7 th day $(N=10)$} & \multicolumn{3}{|c|}{ Healthy group $(\mathrm{N}=11)$} \\
\hline & $\mathrm{R}^{2}$ & r & $P$ & $\mathrm{R}^{2}$ & $\mathrm{r}$ & $P$ & $\mathrm{R}^{2}$ & $r$ & $\mathrm{P}$ \\
\hline \multicolumn{10}{|l|}{ ApEn } \\
\hline RMSSD (ms) & 0.72 & 0.87 & 0.00 & 0.75 & 0.88 & 0.00 & 0.34 & 0.63 & 0.03 \\
\hline RMSM (ms) & 0.35 & 0.65 & 0.03 & 0.43 & 0.70 & 0.02 & 0.45 & 0.71 & 0.01 \\
\hline LFnu & 0.46 & 0.72 & 0.01 & 0.41 & 0.69 & 0.02 & 0.33 & 0.63 & 0.03 \\
\hline HFnu & 0.46 & 0.72 & 0.01 & 0.41 & 0.69 & 0.02 & 0.33 & 0.63 & 0.03 \\
\hline LF/HF ratio & 0.33 & 0.64 & 0.04 & 0.74 & 0.87 & 0.00 & 0.50 & 0.74 & 0.00 \\
\hline
\end{tabular}

$\mathrm{AMI}=$ acute myocardial infarction; $\mathrm{R}^{2}=$ determination coefficient; $r=$ correlation coefficient; $\mathrm{P}=\mathrm{P}$ value; ApEn = approximate entropy; RMSSD = square root of the mean of the sum of the squares of differences between R-R intervals (RRi) divided by the number of RRi in a determined time minus one; RMSM = square root of the sum of the squares of differences of individual values compared to the mean value divided by the number of RRi in a period; $m s=$ milliseconds; $\mathrm{LF}=$ low frequency; $\mathrm{HF}=\mathrm{high}$ frequency; nu = normalized units. The simple linear regression test was used to correlate HRV indexes. 


\section{Discussion}

The results of the linear regression analysis between ApEn and linear methods in time and frequency domains in the present study demonstrate that the values of the coefficient of determination and the correlation coefficient were significant for both the AMI group on the 2nd and 7th day after coronary event and the healthy group. These results differ from the findings of Makikallio et al. (2) who, when comparing HRV in time and frequency domains with ApEn in postAMI patients, observed that the lower the time and frequency domain values, the higher the ApEn values. The differences between our results and those of Makikallio et al. (2) could be attributed to data processing methodology and/or to their decision to interrupt $\beta$-blocker and angiotensin-converting enzyme inhibitor use before the tests. Thus, they reported that AMI contributed to an increase in sympathetic tonus, which was reflected in increased signal complexity, and that the $\beta$-adrenergic system participated little in nonlinear HRV dynamics (24). Nevertheless, we consider that the lack of difference we found between nonlinear ApEn analysis and both time and frequency domain indexes could be attributed to $\beta$-adrenoceptor blockade.

Beckers et al. (24) assessed sympathetic and vagal activity in HR response and observed that after partial pharmacological blockade of the vagal component there was a decrease in the nonlinear complexity of HRV, suggesting that vagal activity is responsible for the introduction of complexity in HR variation.

Krstacic et al. (4) found lower ApEn indexes in post-AMl patients than in healthy individuals and attributed this to the signal's loss of complexity and irregularity due to reduced HRV, increased sympathetic modulation and decreased vagal modulation.

Regarding the comparison of HRV indexes evaluated on the 2 nd and 7 th day after AMI, we found no differences between time and frequency domains or ApEn in our study. This similarity should be considered because of the short evaluation period, i.e., a 5-day interval. Another fact to be considered is the use of drugs, specifically $\beta$-blockers, and
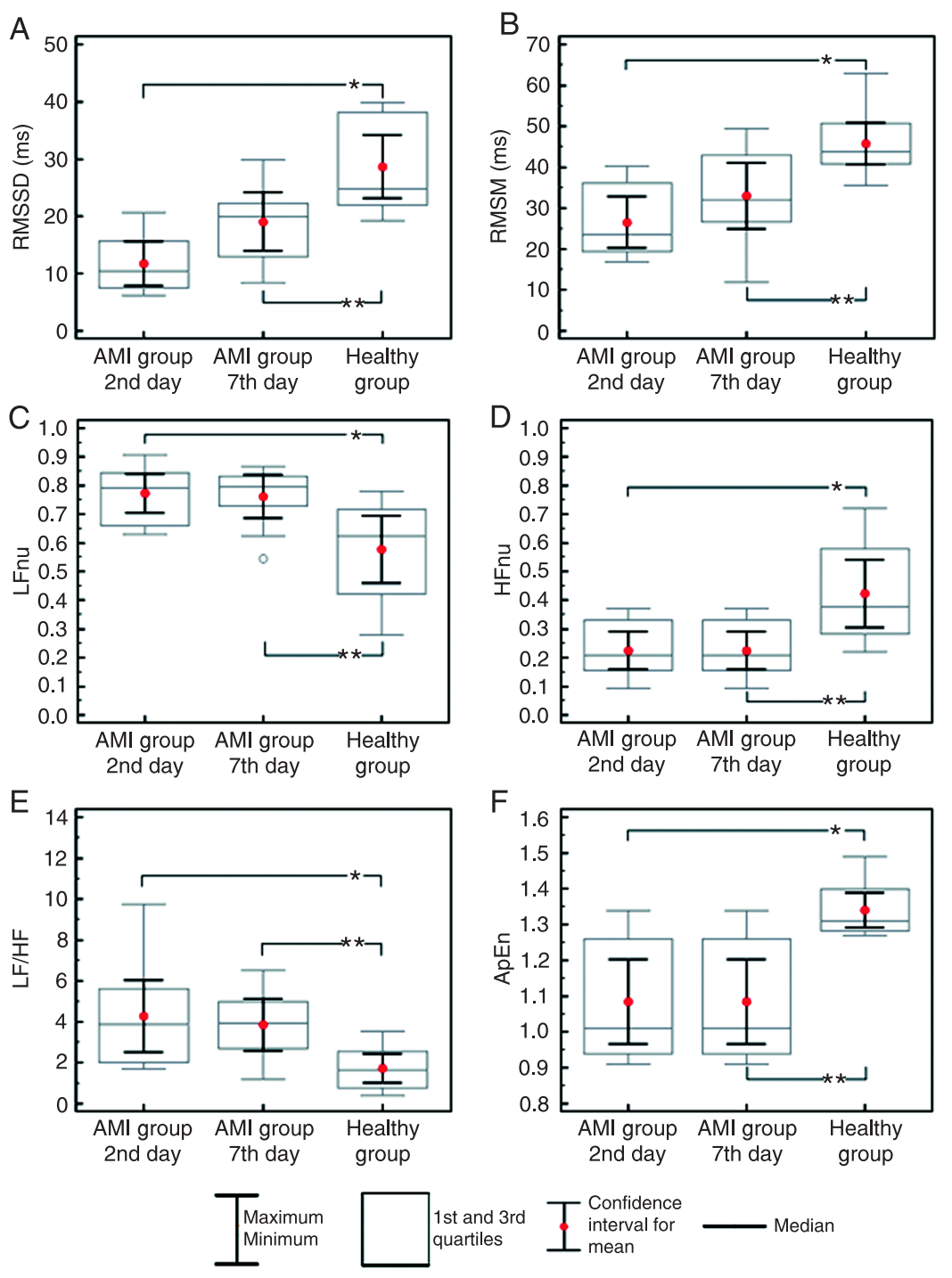

Figure 2. Box plot (median, confidence interval, 1 st and 3rd quartiles, maximum and minimum) showing the results of the time domain analysis [RMSSD $=$ square root of the sum of the squares of differences between RRi divided by the number of RRi in a determined time minus one $(A)$; RMSM = square root of the sum of the squares of differences of individual values compared to the mean value divided by the number of $\mathrm{RRi}$ in a period $(B)]$, the frequency domain analysis [LF = low frequency $(C)$ and HF = high frequency $(D)$ nu (normalized units), and LF/HF ratio $(E)]$ and the nonlinear model analysis $[\mathrm{ApEn}=$ approximate entropy $(F)]$, comparing the acute myocardial infarction (AMI) group on the 2 nd and 7 th day to the healthy group. ${ }^{*} \mathrm{P}<0.05$, AMI group on the 2nd day compared to the healthy group (Mann-Whitney nonparametric test); ${ }^{* *} \mathrm{P}<0.05$, AMI group on the 7th day compared to the healthy group (Mann-Whitney nonparametric test).

the application of chemical or mechanical reperfusion on the first day after AMI.

According to Piegas et al. (16), $\beta$-blockers are first choice medicines for treating AMI since they reduce oxygen demand in the myocardium, HR, BP, and heart contraction. Lampert et al. (25) stated that these drugs, in 
addition to reducing the incidence of morbidity and mortality, act directly on the autonomic nervous system because they specifically antagonize the action of catecholamine on $\beta$-adrenergic receptors and consequently alter HRV indexes by reducing sympathetic activity and promoting parasympathetic recovery.

Considering the effects of $\beta$-blockers on the autonomic modulation of HR, we verified that, even though all patients from the AMI group were taking these drugs, the HRV indexes were similar on the 2nd and 7th day after the coronary event. Similarly, Carpeggiani et al. (26) observed no differences in HRV indexes when comparing the time of admission to and discharge from the hospital.

On the other hand, it has been reported that AMI patients who began the use of $\beta$-blockers between the 5th and 21st day after hospital admission and continued to use them for 6 weeks presented significant increases in time domain (RMSSD) and frequency domain (HF component) indexes, as well as a decrease in the LF/HF ratio (25). These results could be attributed to the period of $\beta$-blocker use, which has effects on the autonomic modulation of HR.

However, it is important to consider that successful chemical or mechanical reperfusion carried out immediately after AMI contributes to the preservation or increase of autonomic function, yielding HRV index values close to the normal range. Nevertheless, it has been observed that autonomic modulation of HR begins to recover from 30 to 60 days after angioplasty (27).

Regarding the coronary reperfusion of patients in the present study, which was carried out on the first day of admission and followed up with HRV analysis on the 2nd and 7th day, the time and frequency domains and ApEn indexes were similar. These results agree with Doulalas et al. (28) and Bonnemeier et al. (29), who reported that HRV indexes are not altered between the 1st and 5th day.

The reduction in the AMI group's HRV indexes compared to those of healthy individuals could be attributed to alterations in autonomic modulation in the sinoatrial node, reflected on lower vagal modulation and higher sympathetic modulation. Previous studies have reported that the alterations in autonomic modulation of HR after an ischemic cardiac event are due to modifications in ionic currents across the cell membrane, leading to electrical instability in the myocytes that promotes increased ventricular work and oxygen demand $(25,27,30,31)$.

It is important to point out that the LFnu (0.79) and LF/ $\mathrm{HF}$ (3.94) values we observed indicated an increase in the sympathetic autonomic modulation of post-AMI patients compared to healthy individuals. Carpeggiani et al. (26) evaluated the HRV response and observed greater LF/HF values in AMI patients who were still in the hospital than in healthy individuals. Similarly, Barbosa et al. (32) observed that patients with cardiac insufficiency also presented important alterations in the sympathetic and parasympathetic systems.

Regarding the ApEn results, our study agrees with Krstacic et al. (4), who observed ApEn values of $0.94 \pm 0.13$ in an AMI group. Moreover, our data are also similar to the results of Acharya et al. (33), who observed ApEn values of 1.68 for healthy, middle-aged individuals.

The intergroup ApEn differences could be attributed to the disease process. In AMI there is a loss of chaotic behavior (nonlinear) in favor of cyclic behavior or lack of variability, which represents a higher risk of abnormality and even mortality (15).

The present study was limited by the difficulty in selecting a sample that would satisfy the proposed inclusion criteria. More detailed studies with larger samples are needed, as well as cross-sectional studies that include patients with AMI who have not received $\beta$-blocker therapy.

\section{Clinical implications}

HRV analysis using linear and nonlinear methods is considered to be important for evaluating and understanding cardiac autonomic modulation in pathological situations like AMI.

In summary, we observed that the HRV of the sample of AMI group patients who used $\beta$-blockers and were treated with coronary reperfusion was similar on the 2 nd and 7 th day. However, upon comparing post-AMI patients with healthy individuals, we observed that patients with AMI presented greater sympathetic modulation and a reduction in vagal modulation. HRV results from linear methods in the time and frequency domains agreed with those obtained by the nonlinear method ApEn. This suggests that linear methods in time and frequency domains and nonlinear methods like ApEn can be employed in the evaluation of the autonomic modulation of HR both in healthy subjects and in post-AMI patients.

\section{Acknowledgments}

The authors would like to thank CNPq for financial support (\#579408/2008-6 to V.C. Kunz, and \#308348/2009-5 and \#478601/2010-7 to E. Silva) and the staff of the Hospital dos Fornecedores de Cana de Piracicaba and Hospital Santa Casa de Misericórdia de Limeira for allowing us to conduct this study. Special thanks are due to Dr. Luciano Domingues Dantas and the patients who participated in this study. 


\section{References}

1. Heart rate variability: standards of measurement, physiological interpretation and clinical use. Task Force of the European Society of Cardiology and the North American Society of Pacing and Electrophysiology. Circulation 1996; 93: 1043-1065.

2. Makikallio TH, Seppanen T, Niemela M, Airaksinen KE, Tulppo M, Huikuri HV. Abnormalities in beat to beat complexity of heart rate dynamics in patients with a previous myocardial infarction. J Am Coll Cardiol 1996; 28: 1005-1011.

3. Oliveira DJ, Gomes MED, Guimarães HN, Aguirre LA. Síntese de sinais de variabilidade da frequência cardíaca baseada em modelagem não-linear. Rev Soc Bras Eng Biomédica 2006; 22: 51-62.

4. Krstacic G, Krstacic A, Smalcelj A, Milicic D, JembrekGostovic M. The "Chaos Theory" and nonlinear dynamics in heart rate variability analysis: does it work in short-time series in patients with coronary heart disease? Ann Noninvasive Electrocardiol 2007; 12: 130-136.

5. Porta A, Guzzetti S, Montano N, Furlan R, Pagani M, Malliani A, et al. Entropy, entropy rate, and pattern classification as tools to typify complexity in short heart period variability series. IEEE Trans Biomed Eng 2001; 48: 1282-1291.

6. Guzzetti S, Borroni E, Garbelli PE, Ceriani E, Della BP, Montano N, et al. Symbolic dynamics of heart rate variability: a probe to investigate cardiac autonomic modulation. Circulation 2005; 112: 465-470.

7. Huikuri HV, Makikallio TH, Peng CK, Goldberger AL, Hintze $\mathrm{U}$, Moller M. Fractal correlation properties of R-R interval dynamics and mortality in patients with depressed left ventricular function after an acute myocardial infarction. Circulation 2000; 101: 47-53.

8. Stein PK, Domitrovich PP, Huikuri HV, Kleiger RE. Traditional and nonlinear heart rate variability are each independently associated with mortality after myocardial infarction. J Cardiovasc Electrophysiol 2005; 16: 13-20.

9. Wallin BG, Charkoudian N. Sympathetic neural control of integrated cardiovascular function: insights from measurement of human sympathetic nerve activity. Muscle Nerve 2007; 36: 595-614

10. Santos-Hiss MD, Melo RC, Neves VR, Hiss FC, Verzola RM, Silva $\mathrm{E}$, et al. Effects of progressive exercise during phase I cardiac rehabilitation on the heart rate variability of patients with acute myocardial infarction. Disabil Rehabil 2011; 33: 835-842.

11. Pantoni CB, Di Thommazo L, Mendes RG, Catai AM, Luzzi $S$, Amaral NO, et al. Effects of different levels of positive airway pressure on breathing pattern and heart rate variability after coronary artery bypass grafting surgery. Braz $\mathrm{J}$ Med Biol Res 2011; 44: 38-45.

12. Perseguini NM, Takahashi AC, Rebelatto JR, Silva E, BorghiSilva A, Porta A, et al. Spectral and symbolic analysis of the effect of gender and postural change on cardiac autonomic modulation in healthy elderly subjects. Braz J Med Biol Res 2011; 44: 29-37.

13. Pincus SM. Approximate entropy as a measure of system complexity. Proc Natl Acad Sci U S A 1991; 88: 22972301.

14. Pincus S. Approximate entropy (ApEn) as a complexity measure. Chaos 1995; 5: 110-117.
15. Pincus S. Aproximate entropy in cardiology. Herzschr Elektrophys 2000; 11: 139-150.

16. Piegas LS, Feitosa G, Mattos LA, Nicolau JC, Rossi Neto JM, Timerman A, et al. Sociedade Brasileira de Cardiologia. Diretriz da Sociedade Brasileira de Cardiologia sobre tratamento do infarto agudo do miocárdio com supradesnível do segmento ST. Arq Bras Cardiol 2009; 93: e179-e264.

17. Ruha A, Sallinen S, Nissila S. A real-time microprocessor QRS detector system with a 1-ms timing accuracy for the measurement of ambulatory HRV. IEEE Trans Biomed Eng 1997; 44: 159-167.

18. Loimaala A, Sievanen H, Laukkanen R, Parkka J, Vuori I, Huikuri $\mathrm{H}$. Accuracy of a novel real-time microprocessor QRS detector for heart rate variability assessment. Clin Physiol 1999; 19: 84-88.

19. Gamelin FX, Berthoin S, Bosquet L. Validity of the polar S810 heart rate monitor to measure R-R intervals at rest. Med Sci Sports Exerc 2006; 38: 887-893.

20. Vanderlei LC, Silva RA, Pastre CM, Azevedo FM, Godoy MF. Comparison of the Polar S810i monitor and the ECG for the analysis of heart rate variability in the time and frequency domains. Braz J Med Biol Res 2008; 41: 854-859.

21. Correa PR, Catai AM, Takakura IT, Machado MN, Godoy MF. [Heart rate variability and pulmonary infections after myocardial revascularization]. Arq Bras Cardiol 2010; 95: 448-456.

22. Malliani A, Pagani M, Lombardi F, Cerutti S. Cardiovascular neural regulation explored in the frequency domain. Circulation 1991; 84: 482-492.

23. Pagani M, Lombardi F, Guzzetti S, Rimoldi O, Furlan R, Pizzinelli $P$, et al. Power spectral analysis of heart rate and arterial pressure variabilities as a marker of sympatho-vagal interaction in man and conscious dog. Circ Res 1986; 59: 178-193.

24. Beckers F, Verheyden B, Ramaekers D, Swynghedauw B, Aubert AE. Effects of autonomic blockade on non-linear cardiovascular variability indices in rats. Clin Exp Pharmacol Physiol 2006; 33: 431-439.

25. Lampert R, Ickovics JR, Viscoli CJ, Horwitz RI, Lee FA. Effects of propranolol on recovery of heart rate variability following acute myocardial infarction and relation to outcome in the Beta-Blocker Heart Attack Trial. Am J Cardiol 2003; 91: 137-142.

26. Carpeggiani C, L'Abbate A, Landi P, Michelassi C, Raciti M, Macerata A, et al. Early assessment of heart rate variability is predictive of in-hospital death and major complications after acute myocardial infarction. Int $J$ Cardiol 2004; 96: 361-368.

27. Soares PP, Moreno AM, Cravo SL, Nobrega AC. Coronary artery bypass surgery and longitudinal evaluation of the autonomic cardiovascular function. Crit Care 2005; 9: R124R131.

28. Doulalas AD, Flather MD, Pipilis A, Campbell S, Studart F, Rizos IK, et al. Evolutionary pattern and prognostic importance of heart rate variability during the early phase of acute myocardial infarction. Int J Cardiol 2001; 77: 169-179.

29. Bonnemeier H, Hartmann F, Wiegand UK, Irmer C, Kurz T, Tolg R, et al. Heart rate variability in patients with acute myocardial infarction undergoing primary coronary angioplasty. 
Am J Cardiol 2000; 85: 815-820.

30. Bigger JT Jr, Fleiss JL, Steinman RC, Rolnitzky LM, Kleiger RE, Rottman JN. Frequency domain measures of heart period variability and mortality after myocardial infarction. Circulation 1992; 85: 164-171.

31. La Rovere MT, Bigger JT Jr, Marcus FI, Mortara A, Schwartz PJ. Baroreflex sensitivity and heart-rate variability in prediction of total cardiac mortality after myocardial infarction.
ATRAMI (Autonomic Tone and Reflexes After Myocardial Infarction) Investigators. Lancet 1998; 351: 478-484.

32. Barbosa PR, Barbosa FJ, de Sa CA. [Effects of age, sex and coronary heart disease on the autonomic modulation of the heart]. Arq Bras Cardiol 1996; 67: 325-329.

33. Acharya UR, Kannathal N, Sing OW, Ping LY, Chua T. Heart rate analysis in normal subjects of various age groups. Biomed Eng Online 2004; 3: 24. 